\title{
Broadband Acoustic Cloak for Ultrasound Waves
}

\author{
Shu Zhang, Chunguang Xia, and Nicholas Fang* \\ Department of Mechanical Science \& Engineering and the Beckman Institute of Advanced Science and Technology, \\ University of Illinois at Urbana-Champaign, Urbana-Champaign, Illinois 61801, USA \\ (Received 12 September 2010; revised manuscript received 9 November 2010; published 10 January 2011)
}

\begin{abstract}
Invisibility devices based on coordinate transformation have opened up a new field of considerable interest. We present here the first practical realization of a low-loss and broadband acoustic cloak for underwater ultrasound. This metamaterial cloak is constructed with a network of acoustic circuit elements, namely, serial inductors and shunt capacitors. Our experiment clearly shows that the acoustic cloak can effectively bend the ultrasound waves around the hidden object, with reduced scattering and shadow. Because of the nonresonant nature of the building elements, this low-loss $(\sim 6 \mathrm{~dB} / \mathrm{m})$ cylindrical cloak exhibits invisibility over a broad frequency range from 52 to $64 \mathrm{kHz}$. Furthermore, our experimental study indicates that this design approach should be scalable to different acoustic frequencies and offers the possibility for a variety of devices based on coordinate transformation.
\end{abstract}

PACS numbers: 43.20.+g, 43.35.+d, 46.40.Ff

Recently, a new design paradigm called coordinate transformation has inspired a series of key explorations to manipulate, store, and control the flow of energy, in the form of either sound, elastic waves, or light radiation $[1,2]$. A set of novel optical devices was proposed based on transformation optics [3-5]; they usually call for complicated medium with anisotropic and spatially varying permittivity and permeability tensor to accomplish the desired functionality. Recent advances in synthetic structured metamaterial [6-8] whose properties are determined by its subwavelength structure, offers the potential to physically implement these complicated media.

Among the most exciting examples is perhaps an electromagnetic cloak that can render the objects invisible. The first experimental demonstration of such a cloak was reported in microwave [9]. However, the invisibility effect was obtained only in a narrow frequency range because of the strong dispersion inherent to the resonant elements used to build the cloak. In addition, such resonances led to undesired material absorption in the cloak. To mitigate these constraints, several different schemes of cloaking utilizing nonresonant structure were proposed [10-14]. One example is a so-called carpet cloak $[15,16]$. However, the waves travel faster through the carpet cloak than through the outer space, such a faster-than-light speed thereby sets a fundamental restriction for broadband application in ambient air.

In contrast, cloaking of other classical waves such as acoustic waves does not suffer from such a limitation for electromagnetic cloaks [17-19]. However, in general the elastodynamic equations do not have this invariance symmetry [20]. Fortunately, acoustic waves in fluids follow such form invariance and several theoretical schemes of transformation have been proposed [21-24]. Theoretical analysis of an acoustic cloak [24] was reported based on the equivalence between transverse electric electromagnetic waves and acoustic waves in a twodimensional [2D] geometry. Yet, this 2D acoustic cloak requires anisotropic mass density which is not common in naturally occurring materials $[25,26]$. Consequently the experimental studies of acoustic cloak have been hampered by the difficulty in creating suitable materials and so far remain challenging.

In this Letter, we overcome the above challenges in acoustic cloak design by introducing an acoustic transmission line approach. The research on acoustic metamaterial was first stimulated by the opportunity to develop artificial media with negative material properties $[27,28]$. By taking the analogy between lumped acoustic elements and electronic circuit elements, this transmission line (TL) approach enabled a new class of acoustic metamaterials [29] and ultrasound focusing through a metamaterial network [30]. As a demonstration, we designed a 2D cylindrical cloak as shown in Fig. 1 in order to hide an object in the center. This acoustic cylindrical cloak is implemented by a 2D array of subwavelength cavities

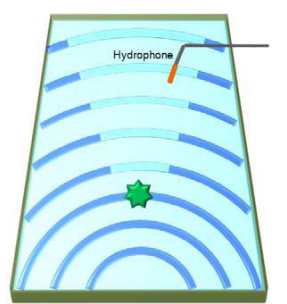

(a)

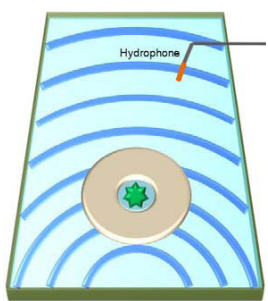

(b)
FIG. 1 (color online). Schematic diagram of the experimental setup. A burst of monotonic signal with a width of 20 periods was used to drive the transducer as an underwater point source in the water tank. One needle-sized hydrophone detected the ultrasonic signals in the immediate environment of (a) the object and (b) the cloaked object. 
and connecting channels with spatially tailored geometry. The acoustic wave propagation through this discrete network can be described by a set of telegrapher's equations in which the motion of the fluid is equivalent to the behavior of the current in the circuit. The acoustic cloak is expected to be low loss and broadband with the use of nonresonant constituent elements.

In our design, the $2 \mathrm{D}$ acoustic metamaterial cloak is designed to squeeze the cylindrical region $0<r<R_{2}$ into an annular region $R_{1}<r^{\prime}<R_{2}$ where $r$ and $r^{\prime}$ are the radial coordinate in the original and transformed system, respectively. The acoustic waves are thus excluded from the extended volume and smoothly bent inside the cloak, with no perturbation of exterior field. We choose the inner and outer radius of the cloak as $R_{1}=13.5 \mathrm{~mm}$, $R_{2}=54.1 \mathrm{~mm}$. Using $2 \mathrm{D}$ telegrapher's equations to describe the distributed acoustic system, this given warping of space can be achieved by providing the desired distribution of the serial inductor and shunt capacitor in the annular region interpreted as $L_{r}=\rho_{w} \frac{\Delta r}{2 S_{r}} L_{\phi}=$ $\rho_{w} \frac{r \Delta \phi}{2 S_{\phi}}\left(\frac{r-R_{1}}{r}\right)^{2} \quad C=2 \Delta r S_{\phi} \beta_{w}\left(\frac{R_{2}}{R_{2}-R_{1}}\right)^{2}$ as plotted in Fig. 2(b). (See the supplementary material [31].) To facilitate the experimental realization of the cloak, here we used the simplified functional form of the cloaking parameters.

The acoustic cloak with the above parameter specification is physically synthesized by a planar network of acoustic circuits machined in an aluminum plate as shown in Fig. 2(a). These building blocks are cascaded in a lattice configuration which is diagonal in a cylindrical basis. In such a topology, the cloak is approximated by 16 homogeneous concentric cylinders.

In our experiment, the water-filled network structure behaves as a lumped anisotropic TL for incoming underwater ultrasound. In each unit cell as shown in Fig. 2(b), as the size of each unit cell is only around one-tenth of the wavelength at operating frequency of $60 \mathrm{kHz}$, the cavity with large volume in center works as an acoustic capacitor $C=\frac{V}{\rho_{w} C_{w}^{2}}$ whereas the channels connecting it to the four neighboring cavities act as serial inductors $L_{r}=\rho_{w} \frac{l_{r}}{S_{r}}$ $L_{\phi}=\rho_{w} \frac{l_{\phi}}{S_{\phi}}$ [32-34]. These relations imply the realization of the TL cloak with the spatially varying parameter profile by tailoring the geometry of the corresponding building blocks as listed in Fig. 2(c). Such an anisotropic circuit network reroutes the paths of underwater sound around the cloaked object without significant scattering. In the lumped circuit model, the aluminum is assumed as acoustically rigid considering the acoustic impedance $\rho c$ of aluminum is around 11 times of that of water. Moreover, the majority of acoustic energy can be predominantly confined in the fluid as a result of the wave excitation in the experiment $[35,36]$.

For experimental confirmation of the cloaking performance, we placed an object in a water tank and compared the wave fronts of propagating ultrasound in our measurement, with or without the presence of our cloak as shown in

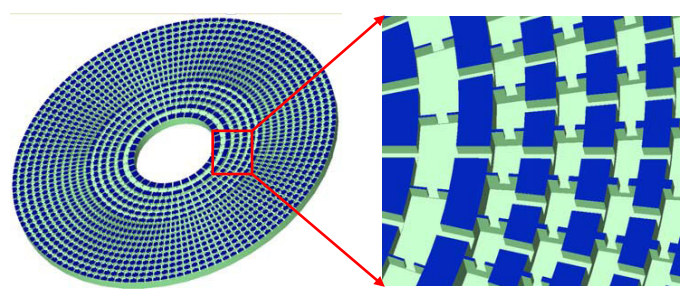

(a)

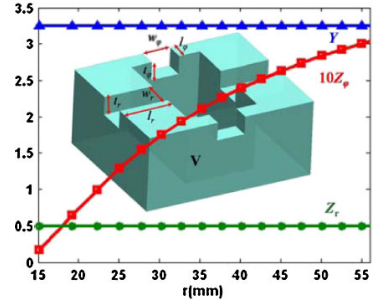

(b)

\begin{tabular}{|c|c|c|c|}
\hline Layer & $l_{r}(\mathrm{~mm})$ & $l_{\varphi}(\mathrm{mm})$ & $V\left(\mathrm{~mm}^{3}\right)$ \\
\hline 1 & 2.05 & 0.10 & 3.00 \\
\hline 3 & 1.37 & 0.22 & 2.29 \\
\hline 5 & 1.24 & 0.41 & 2.06 \\
\hline 7 & 1.24 & 0.30 & 2.06 \\
\hline 9 & 1.24 & 0.41 & 2.06 \\
\hline 11 & 1.24 & 0.52 & 2.06 \\
\hline 13 & 1.24 & 0.63 & 2.06 \\
\hline 15 & 1.24 & 0.74 & 2.06 \\
\hline
\end{tabular}

(c)
FIG. 2 (color online). A 2D acoustic cloak for underwater ultrasound waves. (a) The configuration of the acoustic cylindrical cloak synthesized by an acoustic transmission line, namely, serial inductors and shunt capacitors. The inset is the expanded view of the network. The cavities with large volume work as shunt capacitors and those cavities are connected by narrow channels that act as the serial inductors. (b) One building block of the acoustic circuit, each unit cell consists of one large cavity in the center with channels connecting to the four neighboring blocks. The reduced cloaking parameters are used in the design. The serial impedance $Z_{r}$, shunt admittance $Y$ have constant values and $Z_{\varphi}$ increases as radius changes from $R_{1}=13.5 \mathrm{~mm}$ to the $R_{2}=54.1 \mathrm{~mm}$. (c) The geometry parameters of the building blocks in the layers with odd number are presented in the table. The depth and width $t_{r}, w_{r}$ and $t_{\varphi}, w_{\varphi}$ of the channels along radial and angular directions have constant values of $0.5 \mathrm{~mm}$.

Fig. 1. The object is a steel cylinder with size equal to the inner radius of the cloak. The side of the cloak machined with the metamaterial network was placed against the bottom of the tank in order to seal water inside. The ultrasound waves were launched from a spherical shaped transducer as a point source with a distance of $165 \mathrm{~mm}$ (about 6.5 wavelengths) away from the center of the cloak. To map the pressure field, a hydrophone was mounted on a horizontal linear translation stage to scan in $x-y$ directions. By stepping the hydrophone in small increments of $3 \mathrm{~mm}$ and recording the acoustic pulse signal from the water at every step, we acquired the 2D spatial field distribution of the ultrasound wave scattering pattern.

It is evident in our experiment that the presence of steel cylinder alone in the water tank produces considerable scattering and shadowing at $60 \mathrm{kHz}$ as shown in Fig. 3(a). By surrounding the steel cylinder with the metamaterial cloak in Fig. 3(d), however, the wave trajectory was restored behind the cloak with diminutive distortion in the cylindrical wave fronts, making the cloak and the hidden cylinder invisible under the hydrophone. Very small attenuation of the transmitted fields is observed on the exit 
side of the cloak, demonstrating the low-loss nature of the metamaterial cloak based on transmission line model.

To demonstrate the broadband nature of our designed cloak, the acoustic wave field distributions at 52 and $64 \mathrm{kHz}$ are presented in Figs. 3(b), 3(c), 3(e), and 3(f) for both cases with and without cloak. The field maps from these measurements present similar cloaking behavior with those at $60 \mathrm{kHz}$. This is not surprising since our metamaterial cloak is constructed by nonresonant elements. Theoretically, the cloak is expected to operate over a wide frequency range of 40 to $80 \mathrm{kHz}$. The effectiveness of cloak at high frequency is restricted by two factors. The first is the breakdown of the lumped circuit model when the unit cell is comparable to one quarter of wavelength. The other limit is the cutoff frequency due to the low-pass topology of the circuit network. However, in the current experiment we can only verify the cloaking behavior from 52 to $64 \mathrm{kHz}$ due to the limited operating frequency range of the transducer.

To further quantify the reduction of scattering and shadowing of the cloaked object, we conducted a set of measurements over different frequencies. The peak values of pressure along the wave fronts behind the cloak were obtained using a MATLAB program to process the experimental data. To facilitate the comparison of the cloaking performance, we defined the averaged visibility of an object as $\bar{\gamma}=\frac{1}{n} \sum_{j=1}^{n} \gamma_{j}$, where $\gamma_{j}=\frac{P_{\max , j}-P_{\min , j}}{P_{\max , j}+P_{\min , j}}, P_{\max , j}$ and $P_{\min , j}$ are the maximum and minimum peak values along the wave front numbered by $j$. This can be compared to the traditional measurement of so-called scattering cross-sectional area, but performed for the convenience of the near field measurement and limited field of view in our experiment setup. Figure 4(a) shows one example of the measured peak pressure at $60 \mathrm{kHz}$ along one wave front on the exit side of the object for both cases with and without cloak. This wave front is near the boundary of the cloak between $y=100 \mathrm{~mm}$ and $y=170 \mathrm{~mm}$. As reference, the measurement results for the free space when there are neither objects nor cloak in the water tank is plotted. In Fig. 4(b), the averaged visibility of the cloaked object over all the wave fronts on the exit side is compared with the one with only bare cylinder. The comparison clearly indicates that the cloak preserves the obvious shielding effectiveness over a broad frequency range. We can read the visibility of 0.62 for the bare steel cylinder,

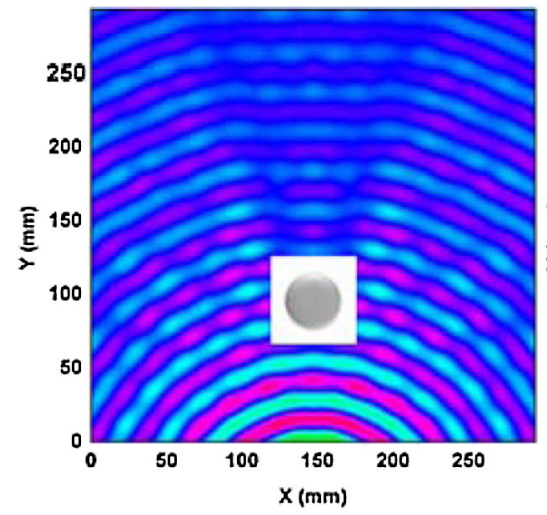

(a)

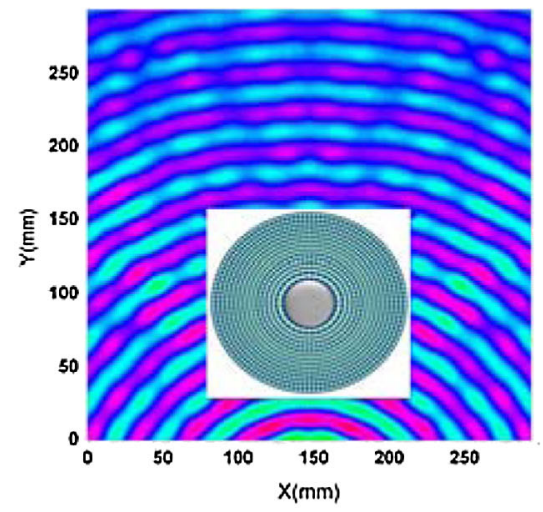

(d)

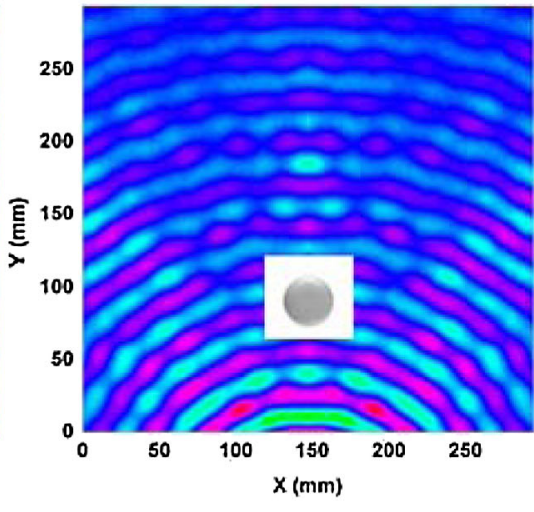

(b)

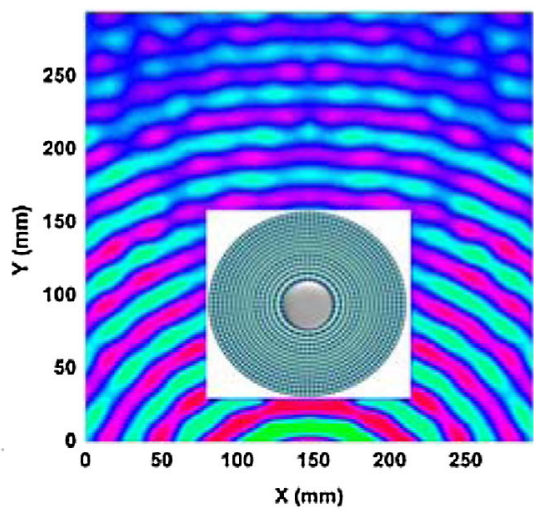

(e)

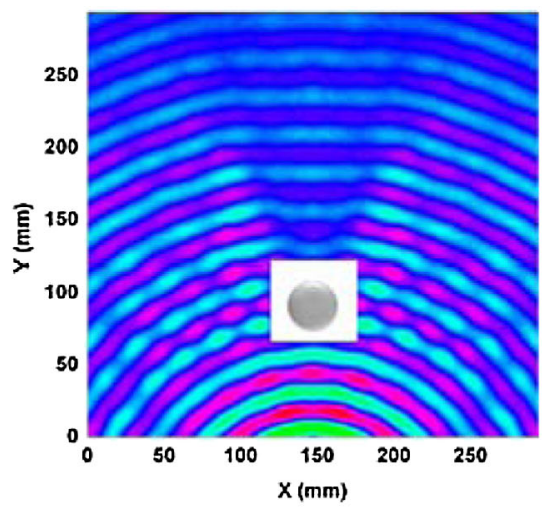

(c)

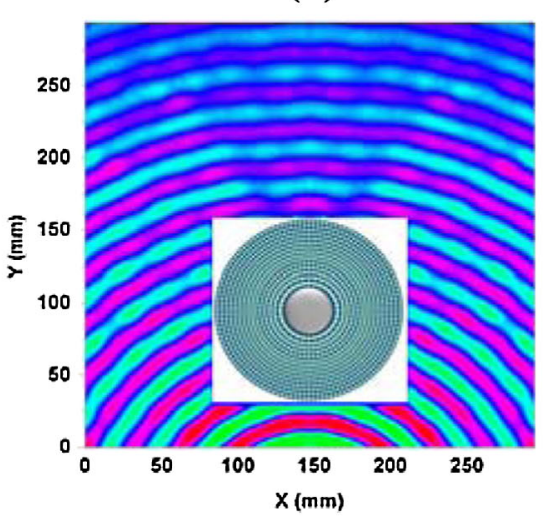

(f)

FIG. 3 (color online). Measured pressure field mappings of the bare steel cylinder and the cloaked steel cylinder illuminated with a point ultrasound source. The cloak lies in the center of the water tank and surrounds the steel cylinder. The scattering field patterns of the bare steel cylinder at (a) $60 \mathrm{kHz}$ (b) $52 \mathrm{kHz}$, and (c) $64 \mathrm{kHz}$. The pseudo-color maps in the immediate environment of the cloaked steel cylinder at (d) $60 \mathrm{kHz}$ (e) $52 \mathrm{kHz}$, and (f) $64 \mathrm{kHz}$. 


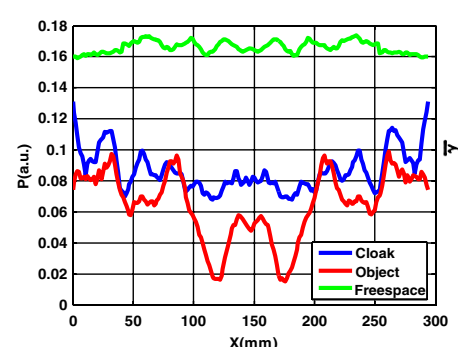

(a)

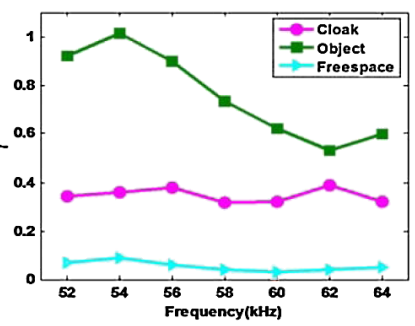

(b)
FIG. 4 (color online). Frequency dependence of the averaged visibility of the steel cylinder with and without the acoustic cloak. (a) The measured peak values of the pressure field along the wave front lies between $y=100 \mathrm{~mm}$ and $y=170$ with and without cloak at $60 \mathrm{kHz}$. The top line plot in green is the reference case when there is no object in the water tank. (b) Plot of the averaged visibility. The experimental results measured with and without cloak are marked by magenta circles and green squares, respectively. The reference visibility when there is no object is marked by blue triangles.

whereas the visibility of the cloaked steel cylinder is reduced to 0.32 at $60 \mathrm{kHz}$, showing the reduction in scattering and shadowing. To make the transmission line approach feasible, we used the reduced cloaking parameter. This leads to the mismatch of impedance between the outer boundary of the cloak and the surrounding water, preventing perfect cloaking performance.

In conclusion, we have demonstrated a $2 \mathrm{D}$ acoustic cloak that can reduce the visibility of the hidden object from underwater acoustic waves. This underwater acoustic cloak can be readily implemented by a network of anisotropic acoustic transmission line. Such a new class of acoustic metamaterial is built from nonresonant circuit elements and can work over a broad frequency range. Moreover, this transmission line approach may have potential applications for a myriad of fascinating devices beyond cloaking based on coordinate transformation.

*nicfang@illinois.edu

[1] J. B. Pendry, D. Schurig, and D. R. Smith, Science 312, 1780 (2006).

[2] U. Leonhardt, Science 312, 1777 (2006).

[3] A. V. Kildishev and V. M. Shalaev, Opt. Lett. 33, 43 (2008).

[4] M. Rahm, S. A. Cummer, D. Schurig, J. B. Pendry, and D. R. Smith, Phys. Rev. Lett. 100, 063903 (2008).

[5] M. Rahm et al., Opt. Express 16, 11555 (2008).
[6] D. R. Smith et al., Phys. Rev. Lett. 84, 4184 (2000).

[7] D. R. Smith, J. B. Pendry, and M. C. K. Wiltshire, Science 305, 788 (2004).

[8] A. Grbic, G. V. Eleftheriades, Phys. Rev. Lett. 92, 117403 (2004).

[9] D. Schurig et al., Science 314, 977 (2006).

[10] W. Cai, U. K. Chettiar, A. V. Kildishev, and V. M. Shalaev, Nat. Photon. 1, 224 (2007).

[11] A. Alù and N. Engheta, Phys. Rev. Lett. 100, 113901 (2008).

[12] U. Leonhardt and T. Tyc, Science 323, 110 (2009).

[13] J. Li and J. B. Pendry, Phys. Rev. Lett. 101, 203901 (2008).

[14] P. Alitalo et al., Appl. Phys. Lett. 94, 014103 (2009).

[15] R. Liu et al., Science 323, 366 (2009).

[16] J. Valentine et al., Nature Mater. 8, 568 (2009).

[17] S. A. Cummer et al., Phys. Rev. Lett. 100, 024301 (2008).

[18] S. Zhang et al., Phys. Rev. Lett. 100, 123002 (2008).

[19] M. Farhat, S. Enoch, S. Guenneau, and A. B. Movchan, Phys. Rev. Lett. 101, 134501 (2008).

[20] G. W. Milton, M. Briane and J. R. Willis, New J. Phys. 8, 248 (2006).

[21] J. B. Pendry and Li Jensen, New J. Phys. 10, 115032 (2008).

[22] H. Chen and C. T. Chan, Appl. Phys. Lett. 91, 183518 (2007).

[23] Y. Cheng, F. Yang, J. Y. Xu, and X. J. Liu, Appl. Phys. Lett. 92, 151913 (2008).

[24] S. A. Cummer and D. Schurig, New J. Phys. 9, 45 (2007).

[25] D. Torrent and J. Sánchez-Dehesa, New J. Phys. 10, 063015 (2008).

[26] D. Torrent and J. Sánchez-Dehesa, New J. Phys. 10, 023004 (2008).

[27] Z. Liu, X. Zhang, and Y. Mao et al., Science 289, 1734 (2000).

[28] L. Feng et al., Phys. Rev. Lett. 96, 014301 (2006).

[29] N. Fang, D. Xi, and J. Xu et al., Nature Mater. 5, 452 (2006).

[30] S. Zhang, L.Yin, and N. Fang, Phys. Rev. Lett. 102, 194301 (2009).

[31] See supplementary material at http://link.aps.org/ supplemental/10.1103/PhysRevLett.106.024301 for the details of the derivation of effective material parameters and experimental setup.

[32] G. W. Stewart, Phys. Rev. 20, 528 (1922).

[33] L. L. Beranek, Acoustics (McGraw-Hill, New York, 1954).

[34] L.E. Kinsler, Fundamentals of Acoustics (Wiley, New York 1982).

[35] C. R. Fuller and F. J. Fahy, J. Sound Vib. 81, 501 (1982).

[36] H. Lamb, in Memoirs and Proceedings of the Manchester Literary \& Philosophical Society (Literary and Philosophical Society of Manchester, London, 1898), Vol. 42, No. 9. 